\title{
Targeted therapy in melanoma
}

\author{
This article was published in the following Dove Press journal: \\ Biologics: Targets \& Therapy \\ 15 October 2009 \\ Number of times this article has been viewed
}

\author{
Hussein Tawbi \\ Neelima Nimmagadda \\ University of Pittsburgh Cancer \\ Institute and the University of \\ Pittsburgh School of Medicine, \\ Pittsburg, PA, USA
}

Correspondence: Hussein Tawbi UPMC Cancer Pavilion, 5150 Centre Avenue, Suite 569, Pittsburgh,

PA 15232, USA

Email tawbih@upmc.edu

\begin{abstract}
Malignant melanoma is a highly lethal disease unless detected early. Single-agent chemotherapy is well tolerated but is associated with very low response rates. Combination chemotherapy and biochemotherapy may improve objective response rates but do not prolong survival and are associated with greater toxicity. Immunotherapeutic approaches such as highdose interleukin-2 are associated with durable responses in a small percentage of patients, but are impractical for many patients due to accessibility and toxicity issues. Elucidations of the molecular mechanisms of carcinogenesis in melanoma have expanded the horizon of opportunity to alter the natural history of the disease. Multiple signal transduction pathways seem to be aberrant and drugs that target them have been and continue to be in development. In this review we present data on the most promising targeted agents in development, including B-raf inhibitors and other signal transduction inhibitors, oligonucleotides, proteasome inhibitors, as well as inhibitors of angiogenesis. Most agents are in early phase trials although some have already reached phase III evaluation. As knowledge and experience with targeted therapy advance, new challenges appear to be arising particularly in terms of resistance and appropriate patient selection.
\end{abstract}

Keywords: targeted therapy, metastatic melanoma, sorafenib, oblimersen, bevacizumab

\section{Introduction}

Malignant melanoma is the most rapidly increasing cancer in incidence in the United States with an estimated 62,480 new cases diagnosed in 2008. In its early stages, melanoma can be surgically cured, leading to five-year survival rates exceeding $90 \%$. However, metastatic disease is uniformly fatal with five-year survival rates less than $2 \%$. Its public health impact is best illustrated in the fact that $50 \%$ of patients dying of melanoma would have lived another 25 years. While adjuvant therapy with high-dose interferon alpha-2b (INF $\alpha-2 b)$ can decrease the risk of recurrence and improve survival in a subset of patients in the adjuvant setting, no available therapy has made any impact on improving survival in the metastatic setting. Dacarbazine (DTIC) remains the only approved chemotherapeutic agent despite modest response rates that do not exceed $10 \%$. Multiple other chemotherapeutic agents, used singly or in combination, have failed to improve on the results obtained with DTIC. Only high-dose interleukin-2 (IL-2) has received US Food and Drug Administration (FDA)-approval based mostly on the achievement of durable responses in 5\% of patients; however IL-2 remains restricted for a select group of patients that can withstand its toxicities and can only be delivered in specialized settings. There is an urgent need for new agents that can alter the natural history of melanoma and improve response rates and survival. 
The era of targeted therapies, which was heralded by a better understanding of tumor biology, offers significant promise in the treatment of melanoma but also presents new challenges. In this review, the most prominent targeted agents investigated in melanoma therapy will be presented with emphasis on future directions in which patient selection and combination therapy will likely become standard.

\section{Sorafenib}

\section{Mechanism of action}

Sorafenib is FDA-approved for the treatment of advanced primary renal cell carcinoma and advanced primary hepatocellular carcinoma. It is an oral multikinase inhibitor that inhibits tumor growth by acting on the tumor cells and cells of the tumor vasculature. It inhibits tumor cell proliferation by targeting the mitogen-activated protein kinase (MAPK) pathway at the level of Raf kinase and/or induces tumor cell apoptosis. Sorafenib also potently inhibits vascular endothelial growth factor receptor (VEGFR)-1, -2 and -3 , and platelet-derived growth factor receptor (PDGFR)- $\beta$ tyrosine kinase autophosphorylation. These proangiogenic receptor tyrosine kinases (RTKs) signal through Raf/MEK/ERK to induce proliferation and prolong the survival of vascular endothelial cells, which form new blood vessels. The proangiogenic RTKs also promote the proliferation, survival, and recruitment of pericytes, by signaling through Raf, resulting in the stabilization of newly formed blood vessels. ${ }^{1}$

$B R A F$ is an activating oncogenic mutation found in $60 \%$ of melanomas. V600E is the most prevalent mutation in $B R A F$. Most of the transforming activity of the BRAF V600E occurs through the activation of the MAPK pathway. ${ }^{2}$ Somatic V600E mutated BRAF showed an increased sensitivity to (MEK) inhibition and resulted in more potent tumor cell growth arrest than those cell lines not expressing the V600E mutation. Regardless of BRAF V600E mutational status, virtually all melanomas have activity in the MAPK pathway. $^{3}$

Sorafenib's multiple targets, including Raf- $1,{ }^{4,5}$ wild-type $B R A F$, oncogenic b-raf V600E, and proangiogenic RTKs, ${ }^{6}$ enable its action on tumor cells as well as on tumor vasculature to induce apoptosis and inhibit proliferation as well as angiogenesis in preclinical models. ${ }^{7-9}$ This provides sorafenib with the potential for activity against a wide variety of tumor types. The targeting of multiple Raf isoforms and RTKs by sorafenib may also provide a means to overcome multidrug resistance. Expression of the multidrug resistance $1(m d r-1)$ gene is associated with solid tumors that have a high level of intrinsic or acquired chemoresistance. ${ }^{10}$
In addition, downregulation of antiapoptotic molecules may help sensitize tumor cells to chemotherapy. The inhibition of tumor angiogenesis can therefore enhance delivery of cytotoxic agents by affecting the vasculature, and increase the effectiveness of concomitantly administered chemotherapy.

\section{Safety profile}

Single-agent sorafenib at $400 \mathrm{mg}$ twice daily (bid) was found to be generally well tolerated in phase I-III clinical trials. The majority of adverse events were mild to moderate in severity, followed a predictable course, and were manageable. The most frequently reported drug-related adverse events were dermatologic (hand-foot skin reaction [HFSR], rash/ desquamation, gastrointestinal [diarrhea], and constitutional [fatigue]). ${ }^{11-13}$ Severe biochemical abnormalities, myelosuppression, hematologic, cardiovascular, hepatic, and renal toxicities were rarely reported. ${ }^{12}$ Interestingly, treatmentemergent hypertension was observed in $5 \%-17 \%(<5 \%$ at Grade 3 or 4) of patients receiving sorafenib $400 \mathrm{mg}$ bid and was readily manageable with antihypertensives. ${ }^{11,12}$

The favorable safety profile of sorafenib on a continuous administration schedule and its convenient oral route of administration suggests that it could be combined with other antitumor agents, including cytotoxic agents that are usually associated with higher levels of toxicity, as well as other targeted therapies. ${ }^{14}$

\section{Clinical experience in melanoma}

The effects of the combination of paclitaxel, carboplatin, and sorafenib have been investigated in a phase I/II trial of 35 patients with progressive stage IV melanoma pretreated with no more than three previous chemotherapy regimens. The preliminary results showed a high rate of partial response (PR) $40 \%$ and stable disease (SD) $43 \%$, but the antitumor activity was independent of b-raf mutational status. Responses were observed mainly in patients with skin, subcutaneous, and lymph node metastases (stage M1a) and a limited number of previous therapies. This has lead to two large multicenter randomized placebo-controlled phase III trials investigating the addition of sorafenib to a carboplatin and paclitaxel backbone in both first- and second-line therapy of metastatic melanoma. Both trials have been concluded although results from the first-line trial have not been released yet. The PRISM trial was a phase III, randomized, double-blind, placebo-controlled study conducted to evaluate the efficacy and safety of sorafenib with carboplatin and paclitaxel (CP) in patients with advanced melanoma who had progressed on a DTIC or temozolomide (TMZ)-containing 
regimen (second line setting). A total of 270 patients were randomly assigned to each arm. The median progression-free survival (PFS) was 17.9 weeks for the placebo plus CP arm and 17.4 weeks for the sorafenib plus CP arm (hazard ratio, $0.91 ; 99 \%$ confidence interval [CI]: 0.63 to 1.31 ; two-sided $\log$-rank test, $P<0.49$ ). Response rate was $11 \%$ with placebo versus $12 \%$ with sorafenib. The addition of sorafenib to $\mathrm{CP}$ did not improve any of the end points over placebo plus $\mathrm{CP}$ and cannot be recommended in the second-line setting for patients with advanced melanoma. Both regimens had clinically acceptable toxicity profiles with no unexpected adverse events. ${ }^{15}$

Sorafenib was also evaluated in combination with DTIC in a single-center, open-label, phase I, dose-escalation trial in patients with metastatic melanoma. ${ }^{16}$ Among 18 evaluable patients, three (17\%) had PR and $11(61 \%)$ had SD. This combination was further evaluated in clinical trials, including a phase II open-label, first-line, uncontrolled study as well as a phase II randomized, placebo-controlled study in patients with unresectable stage III or IV melanoma. In the uncontrolled phase II study, sorafenib and DTIC were well tolerated and yielded promising efficacy results in these patients with a poor prognosis. Eight patients (10\%) achieved PR and 34 (41\%) had SD; the median PFS duration was 14 weeks and the median overall survival (OS) time was 41 weeks. $^{17}$ These data are encouraging, compared with DTIC alone, which achieved a response rate of $7.5 \%$ and a PFS time of six weeks. Results from a placebo-controlled study support a better efficacy trend in terms of objective responses and PFS compared with DTIC alone in advanced melanoma. The median PFS times were 21.1 versus 11.7 weeks for sorafenib in combination with DTIC compared with DTIC plus placebo, respectively. ${ }^{18}$

Sorafenib has been evaluated in combination with TMZ, an oral alkylating agent approved for the treatment of patients with metastatic melanoma with or without brain metastases. Results from a four-arm phase II trial demonstrated encouraging antitumor activity and tolerability of this combination in patients with metastatic melanoma. An overall response rate of $19 \%$ was observed in 78 patients across two arms of the study. ${ }^{19}$

Overall, the most promising sorafenib-based combination approach appears to involve DTIC, which produced a fairly consistent level of preliminary responses or SD in patients with advanced melanoma. ${ }^{17,18,20}$

While the development of sorafenib has been fraught with difficulties, in some respects related to selectivity, novel BRAF kinase inhibitors that are selective for the oncogenic
V600E mutant are showing exciting results. A phase I trial of PLX4032, an oral, selective inhibitor of the V600E mutant $B R A F$ enrolled 54 patients, most with metastatic melanoma. A change in formulation was to improve bioavailability after around half the patients were enrolled and the maximum tolerated dose of the new formulation was determined to be $720 \mathrm{mg}$ bid. Of the melanoma patients, five of the seven with $B R A F$ V600E treated at $>240 \mathrm{mg}$ bid had tumor regression, with one confirmed PR and one unconfirmed PR; two of four patients with unknown V600E status had tumor regression one reaching a PR; two $B R A F$ wild-type patients had progressive disease. Three thyroid cancer patients with V600E mutations had tumor regression (range 9\%-16\%) that did not reach PR. The main dose-limiting toxicities included rash, fatigue, elevated liver enzymes, and pancytopenia. PLX4032 appears to be a very promising novel and specific mutant BRAF kinase inhibitor that may pave the way for more effective targeted agents. ${ }^{21}$

\section{Oblimersen \\ Mechanism of action}

Oblimersen (OBL) is a Bcl-2 antisense oligonucleotide that selectively targets Bcl-2 RNA for degradation by RNase $\mathrm{H}$ thereby decreasing $\mathrm{Bcl}-2$ protein production. The $\mathrm{Bcl}-2$ protein, which is a potent inhibitor of apoptosis, is overexpressed in many cancers, including follicular lymphomas, breast, colon and prostate cancers, and intermediate-/highgrade lymphomas. By reducing the amount of $\mathrm{Bcl}-2$ protein in cancer cells, OBL may enhance the effectiveness of conventional anticancer treatments. ${ }^{22}$

\section{Clinical experience in melanoma}

In a phase I/IIa trial in which OBL was administered twice daily by subcutaneous injection to patients with metastatic melanoma, patients also received DTIC on day 5 of each week. Six of 14 evaluable patients (43\%) experienced antitumor responses, including a pathologically confirmed response in an elderly patient. Two additional patients had SD. The median survival duration had exceeded one year by the time the study was reported. In 10/12 (83\%) evaluable patients, OBL treatment caused the downregulation of Bcl-2 protein, with the maximum response occurring at day 5 of treatment. $^{23}$

Similarly, in a dose-escalating study of an infusion regimen of OBL in combination with DTIC in 14 patients with stage IV metastatic melanoma, most patients had reductions from baseline in Bcl-2 protein levels in cutaneous melanoma metastases. Antitumor activity was observed 
in six patients, including one complete response, two $\mathrm{PR}$ and two minor responses with $\mathrm{SD}$ for $\geq 1$ year. The estimated median survival duration at the time of the report was $>1$ year. Patients received a 14-day continuous intravenous infusion of OBL $0.6-6.5 \mathrm{mg} / \mathrm{kg} /$ day plus intravenous DTIC $200 \mathrm{mg} / \mathrm{m}^{2} /$ day on days 5-9 of OBL administration. Treatment cycles could be repeated at 28-day intervals in patients who were stable or who experienced a response. ${ }^{24}$

An OBL-TMZ-albumin-bound paclitaxel (ABX) combination was synergistic in the preclinical setting, and a phase I/II study of this combination was initiated in patients with metastatic melanoma and normal lactate dehydrogenase (LDH). TMZ is an orally administered chemotherapy with a mechanism of action and efficacy in melanoma similar to that of DTIC. ABX is currently being explored in melanoma. Chemotherapy-naïve patients with advanced melanoma, baseline $\mathrm{LDH} \leq 1.1 \times$ upper limit of normal (ULN), and measurable disease were enrolled in the study. Treatment included OBL $7 \mathrm{mg} / \mathrm{kg} / \mathrm{d}$ (continuous intravenous infusion, days $1-7$ and 22-29), TMZ $75 \mathrm{mg} / \mathrm{m}^{2} / \mathrm{d}$ (days $1-42$ ), and ABX $175 \mathrm{mg} / \mathrm{m}^{2}$ (days 7 and 29) in cohort 1 and $260 \mathrm{mg} / \mathrm{m}^{2}$ in cohort 2 for four 56-day cycles. All 14 (1-M1a, 5-M1b, 8-M1c) patients planned for cohort 1 have been enrolled; treatment is ongoing in 10. Four patients have achieved a PR ( $>50 \%$ tumor reduction) lasting more than two cycles (range: 3-6 cycles); two with SD lasting three cycles; and two with PD after one cycle. In six patients it is too early to assess response. OBL and TMZ have no effect on ABX pharmacokinetics. Tumor and serologic assessment of biomarkers correlate with clinical and radiologic responses or progression. Prolonged clinical activity has been seen and the regimen is safe and well tolerated. Cohort 2 is open to enrolment. ${ }^{25}$

A phase III study (NCT00016263) of OBL in combination with DTIC was conducted in patients with malignant melanoma. Patients were randomly assigned to receive DTIC alone or in combination with OBL. The addition of OBL to DTIC improved median overall survival, compared with that in patients treated with DTIC alone (9.0 vs 7.8 months); however, the difference between the groups was not significant $(P=0.077)$. In addition, the analyses indicated that a blood test (LDH) could be used to identify patients who were likely to derive the most benefit from OBL therapy. Baseline LDH was highly predictive of OBL effect in melanoma independently of tumor burden; this suggested that elevated LDH may reflect aggressive tumor biology. After a minimum followup duration of 24 months, patients treated with OBL plus DTIC versus DTIC alone yielded significantly higher overall response rates $(13.5 \%$ vs $7.5 \%, P=0.007)$, complete responses (2.8\% vs $0.8 \%, P=0.03)$, durable responses $(7.3 \%$ vs $3.6 \%$, $P=0.03)$ and median PFS (2.6 vs 1.6 months, $P=0.0007){ }^{26}$ In summary, the use of OBL with DTIC can improve multiple outcomes in patients with advanced melanoma particularly those with normal base line LDH.

The most common side effects of OBL include nausea, vomiting, pyrexia, fatigue, anorexia, thrombocytopenia, neutropenia, and anemia. OBL-associated thrombocytopenia did not cause an increase in serious bleeding, and the incidence of neutropenic fever was low. ${ }^{26}$

\section{Bevacizumab}

\section{Mechanism of action}

Bevacizumab is FDA-approved for use in metastatic colorectal cancer, non-small cell lung cancer, and metastatic breast cancer. It is a recombinant, humanized $\mathrm{Ab}$ targeted against VEGF-A and blocks its binding to the VEGF receptors. VEGF regulates vascular proliferation and induces permeability, both of which are important for tumor growth. It also has antiapoptotic activities in newly formed endothelial cells. The expression of VEGF in tumor cells is stimulated by hypoxia, hypoglycemia, Ras antigen, and inactivation of tumor suppressor gene p53/von Hippel-Landau gene. Melanoma cells with a nonaggressive phenotype when exposed to chemotherapy acquire a much more tumorigenic and metastatic phenotype. ${ }^{27}$ This is in part through overproduction of VEGF, which may render both endothelial and cancer cells resistant to chemotherapy through a variety of mechanisms: 1) enhancement of tumor growth through induction of angiogenesis; 2) impairment of delivery of chemotherapy to the tumor through increase in interstitial fluid pressure; ${ }^{28} 3$ ) protection of tumor-associated endothelial cells against cytotoxicity; ${ }^{29}$ and 4) initiation of autocrine survival signals in cancer cells. ${ }^{30,31}$ In comparison with conventional chemotherapy, the antiangiogenic effects of bevacizumab are indirect and not necessarily lethal. ${ }^{32}$ However, the addition of bevacizumab to conventional chemotherapy has been shown to control tumor growth and progression more effectively than chemotherapy alone in patients with certain solid tumors. ${ }^{33,34}$ This probable explanation lies in bevacizumab's ability to dampen the effects of VEGF upregulation induced by chemotherapy.

\section{Safety profile}

The most common adverse events for bevacizumab were neutropenia, anemia, thrombocytopenia, fatigue, nausea/ vomiting, neuropathy, hypertension, bleeding episodes, 
thrombotic events, and proteinuria ranging in severity from clinically silent to nephritic syndrome. Further studies are required to determine the relative value of bevacizumab in combination with chemotherapy. ${ }^{35}$

\section{Clinical experience in melanoma}

A randomized phase II trial of bevacizumab with or without daily low-dose INF $\alpha$-2b in metastatic malignant melanoma. Patients exhibiting a clinical response or stable disease after 12 weeks were treated until disease progression. Thirty-two patients (16 per arm) were accrued. Eight patients (five bevacizumab, three bevacizumab plus INF $\alpha-2 b$ ) had prolonged disease stabilization (24 to 146 weeks). Plasma levels of VEGF and fibroblast growth factor (FGF) did not correlate with any clinical parameter. The patient with the longest period of stable disease had the highest baseline VEGF and FGF. Bevacizumab was well tolerated and prolonged disease stabilization was achieved in one-quarter of metastatic melanoma patients. Low-dose IFN $\alpha-2 b$ did not augment the activity of bevacizumab. ${ }^{32}$

A two-stage phase II clinical trial was conducted in patients with unresectable stage IV metastatic melanoma to assess antitumor activity and the toxicity profile of the combination of carboplatin, paclitaxel $\left(80 \mathrm{mg} / \mathrm{m}^{2}\right.$ intravenous on days 1,8 , and 15$)$, and bevacizumab (10 $\mathrm{mg} / \mathrm{kg}$ intravenous on days 1 and 15). Treatment was continued until progression or intolerable toxicity. Fifty-three patients (62.3\% male) were enrolled. Nine (17\%) patients achieved PR, and another 30 (57\%) achieved SD for at least eight weeks. Median PFS and median OS were 6 and 12 months, respectively. This combination of carboplatin, paclitaxel, and bevacizumab appears to be moderately well tolerated and clinically beneficial in patients with metastatic melanoma. ${ }^{35}$

\section{mTOR inhibitors \\ Mechanism of action}

Rapamycin (sirolimus) is an immunosuppressive drug with antiproliferative activity. CCI-779 is an ester analog of rapamycin with improved aqueous solubility and pharmacokinetic properties (432917). Drugs of this class inhibit cell cycle progression by binding to FK506-binding protein-12 (FKBP12) to form a complex that interacts with the "mammalian target of rapamycin" (mTOR), resulting in inhibition of signal transduction pathways required for progression through the cell cycle. ${ }^{36}$ Inhibition of mTOR suppresses $\mathrm{p} 70 \mathrm{~s} 6 \mathrm{k}$ activity, decreasing translation of elements of the translational machinery. Inhibition of mTOR by rapamycin/CCI-779 also prevents cyclin-dependent kinase activation, inhibits phosphorylation of retinoblastoma protein as well as accelerates the turnover of cyclin D1 that leads to a deficiency of active cdk4/cyclin D1 complexes, all of which can inhibit cell cycle kinetics at the G1/S phase transition (439022), (439028). Perturbation of these pathways, downstream of protein kinase B ( $\mathrm{PKB} / \mathrm{AKT}$ )/phosphoinositol-3-kinase $(\mathrm{PI} 3 \mathrm{~K})$ activities, results from upstream mutations, that are mutated in human cancers, most commonly loss of the PTEN (phosphatase and tensin homolog deleted on chromosome 10) tumor suppressor, which leads to overexpression of AKT or PI3K resulting in $\mathrm{mTOR}$ overexpression. PTEN mutations are found in approximately one-half of melanomas. ${ }^{37}$ The resulting increase in the activity of mTOR is believed to "uncouple" it from the normal control exerted on its activity by the availability of nutrients required for cell proliferation. ${ }^{38}$

Elucidation of the therapeutic mechanisms of antitumor activity for CCI-779 and other mTOR inhibitors has led to an increased understanding of the mechanisms of resistance, which include mutations in mTOR, FKBP12, or any of the many others associated with mTOR-related pathways. ${ }^{39}$

\section{Safety profile}

The dose-limiting toxicities for CCI-779 included myelosuppression, diarrhea, stomatitis, fever, fatigue, and hyperlipidemia. Skin reactions were common, ranging from dry desquamation to eczematoid, urticarial, and acneiform rash. Acute infusion reactions resembled histamine-release phenomena and have been avoided by premedication with antihistamines. ${ }^{40,41}$ Based on these safety data as well as pharmacokinetic and pharmacodynamic results, a fixed dose of $250 \mathrm{mg}$ was proposed for evaluation on a weekly dosing schedule in patients with advanced melanoma. The spectrum of toxicities associated with this agent overlaps with those of several other drugs in routine use, which limits the choices of combinations that could be safely considered. The optimal use of this class of agents in malignancy will await the design of regimens that take into account the need for nonoverlapping clinical toxicities and complementary antitumor mechanisms that minimize the emergence of resistant clones.

\section{Clinical experience in melanoma}

The results of phase I trial on CCI-779 did not have sufficient antitumor activity in melanoma to warrant further evaluation as a single agent. A preliminary report in 2003 details the results of a phase I dose-escalation study that combined CCI-779 with IFN- $\alpha$ at a low dose, which could be escalated in the individual patient if no dose-limiting toxicity of CCI-779 occurred. The authors concluded that the 
combination was well tolerated and potentially active, with mechanisms of action that included both direct antitumor activity and antiangiogenic effects. ${ }^{42}$

\section{Bortezomib Mechanism of action}

Bortezomib (PS-341) is a dipeptidyl boronic acid analog, which is a potent and reversible proteasome inhibitor. Cells exposed to PS-341 accumulate in the G2-M phase of the cell cycle, and some undergo apoptosis. ${ }^{43,44}$ The ubiquitin-mediated proteasome pathway regulates a group of intracellular proteins that govern cell cycle, tumor growth, and survival. This pathway is the principal mechanism of degradation for short-lived cellular regulatory proteins, including $\mathrm{p} 53,{ }^{45}$ cyclins and the cyclin-dependent kinase inhibitors p2 $1^{46}$ and p27, the estrogen receptor ${ }^{47}$ and the inhibitor (IKB) of nuclear transcription factor

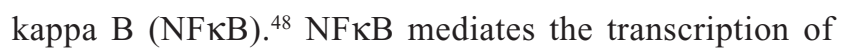
proteins including VEGF and the cell adhesion molecules (CAM) E-selectin, intracellular cell adhesion molecule-1 (ICAM-1), and vascular cell adhesion molecule-1 (VCAM-1), implicated in angiogenesis and tumor metastasis in vivo, and regulates the inhibition of tumor necrosis factor-alpha (TNF- $\alpha$ )-mediated cell death. ${ }^{49}$ Altered degradation of cell cycle control proteins can result in accelerated and uncontrolled cell division and thereby promote cancer growth and spread. Central to the pathway is the $26 \mathrm{~S}$ proteasome, an adenosine triphosphate-dependent, multicatalytic protease that selectively degrades polyubiquitinated proteins. Normal activity of the $26 \mathrm{~S}$ proteasome rapidly clears polyubiquitinated proteins from the cell. While its inhibition permits accumulation of substrate polyubiquitinated proteins and the disruption of cell cycle checkpoints and apoptosis pathways, and thus offers a promising approach for the treatment of malignancies. Preclinical data point to the role of PS-341-induced chemosensitization and radiosensitization. ${ }^{50}$

\section{Safety profile}

PS-341 can be safely administered as an intravenous bolus without premedication, and is generally very well tolerated. The major toxicities were constipation, ileus, sensory neuropathy, thrombocytopenia, fatigue, abdominal pain, infection without neutropenia and malaise. Caution should be exercised with PS-341 in patients with pre-existing peripheral neuropathy. ${ }^{51}$

With its unique mechanism of action and the lack of significant myelosuppression, one can anticipate an eventual role in cancer treatment for PS-341 and newer proteasome inhibitors. The rationale for drug combinations with nonneurotoxic drugs has been advanced and such trials are ongoing in phase I and phase II studies. It is also possible that potentially neurotoxic drugs might be safely combined with PS-341 without undue problems if the dose of these agents is kept below a threshold associated with a high probability of inducing sensory neuropathy. Additional mechanistic studies will be required to facilitate additional progress in the integration of PS-341 in cancer therapeutics.

\section{Clinical experience in melanoma}

A phase I study of PS-341 and INF $\alpha-2 b$ in patients with metastatic melanoma with the primary objective of determining the safety, tolerability and dose limiting toxicities of the combination therapy showed that PS-341 and IFN can be safely administered in patients with treated CNS metastasis. Toxicities were similar to IFN alone. Antitumor response to slowly proliferating tumors are limited. Accrual continues to the highest dose level. ${ }^{52}$

A phase I trial of DTIC and PS-341 in melanoma and soft tissue sarcoma showed that the recommended phase II doses will be at least DTIC $250 \mathrm{mg} / \mathrm{m}^{2}$ and PS-341 $1.6 \mathrm{mg} / \mathrm{m}^{2}$, and dose escalation continues. Treatment has been generally well tolerated with known DTIC and PS-341 toxicities. Among eight melanoma patients, there is one partial PR, one with SD on treatment for 15 weeks, six with progressive disease at $6,7,8,8,13$, and 13 weeks, respectively. At full dose PS-341 and moderate dose DTIC for this schedule, this regimen was feasible and generally well-tolerated. Activity beyond that of DTIC alone is not apparent to date, but several or all patients have been treated at less than maximum tolerated doses. ${ }^{53}$

In a phase II study of PS-341 in the treatment of metastatic malignant melanoma, PS-341 was administered at $1.5 \mathrm{mg} / \mathrm{m}^{2}$ intravenous bolus twice weekly for two weeks out of every three weeks. Twenty-six patients were evaluable for toxicity, of which 10 patients $(42 \%)$ had grade 3 toxicities. This study demonstrated that single agent PS-341 is ineffective and toxic in metastatic melanoma patients at the dose and schedule used in this trial. ${ }^{51}$

\section{MEK inhibitors}

\section{Mechanism of action}

MEK is a tyrosine kinase downstream of B-Raf in the MAPK pathway. In the aberrant activation of MAPK pathway, a mitogen signal transduction pathway provides proliferative advantage to tumor cells. The binding of growth factors induces receptor dimerization and autophosphorylation on 
tyrosine residues. This exchange elicits a conformational change in Ras, a membrane localized protein. This change enables it to bind to Raf-1 and recruit it from the cytosol to the cell membrane, where Raf-1 activation takes place. Activated Raf-1 phosphorylates and activates MEK, which in turn phosphorylates and activates extracellular signalregulated kinase (ERK). Activated ERK has many substrates in the cytosol (eg, cytoskeletal proteins, phospholipase $\mathrm{A}_{2}$, and signaling proteins, including tyrosine kinase receptors, estrogen receptors, SOS, STAT proteins, and others). ERK can enter the nucleus to control gene expression by phosphorylating transcription factors, leading to overproduction of components involved in angiogenesis and tumor progression. $^{54}$

As the melanomas progress, there is functional redundancy between the numerous signaling pathways. It was shown that targeting either the PI3K or MEK pathway alone led to cytostasis and was associated with a reversible G1-phase cell cycle arrest. ${ }^{55,56}$ The MEK inhibitor AZD6244 led to the stabilization of established human melanoma xenografts, but not tumor regression, ${ }^{55}$ suggesting that the effects of MEK inhibition in this setting were largely cytostatic. Lack of good cytotoxic activity after either MEK or PI3K inhibition alone suggests that multiple signaling pathways need to be targeted simultaneously to induce melanoma regression.

The current expectation is that BRAF inhibitors will be used in combination with either other targeted therapy agents or established chemotherapy regimens. However, it is necessary to rigorously evaluate each of the novel BRAF and MEK inhibitors as single agents so that we understand their distinct pharmacological properties and their respective abilities to hit their target and perturb proliferation or cell viability. Only with this knowledge can we make an informed selection of agents for further development.

\section{Safety profile}

The safety profile of MEK inhibitors has not been optimal as dose-limiting diarrhea and rash possibly preclude the delivery of a dose required to adequately suppress the MAPK pathway. Phase II trials of this drug were suspended because of the occurrence of retinal vein thrombosis in several patients.

\section{Clinical experience in melanoma}

The MEK inhibitors - PD0325901 and AZD6244 - have progressed through phase I, and in the case of AZD6244, phase II clinical trials. PD0325901 has been evaluated in a phase I trial, in which most of the patients had melanoma. Of these patients, two out of 27 experienced an objective response $(\mathrm{OR})$ and five additional patients showed some SD. AZD6244 was evaluated in a phase I trial among patients with advanced solid tumors. ${ }^{57}$ Of the melanoma patients treated, none had an OR; however, four patients maintained SD for more than six months, suggesting a clinical benefit.

A randomized phase II trial was completed among 200 patients with melanoma. Patients were randomized in a 1:1 manner to AZD6244 or temozolomide. ${ }^{58}$ Five ORs were observed among 42 patients with $B R A F$ V600E mutations ( $12 \%$ OR rate), indicating that a subset of $B R A F$ mutant melanomas may be sensitive to this agent. As there was no improvement in PFS compared with single-agent temozolomide, AZD6244 was deemed insufficient to warrant further single-agent clinical trials in melanoma.

As the preclinical data support the selective activity of RAF and MEK inhibitors in BRAF mutant melanoma, stratifying the correct patient population for clinical trials is imperative for designing effective and informative clinical trials. This also requires real-time mutation screening as part of the clinical trial. If a responsive subset of $B R A F$-mutated melanomas can be identified, it may be possible to rapidly develop RAF and MEK inhibitors as single agents for this population, whereas combination strategies would be explored for the remaining.

\section{Thalidomide Mechanism of action}

On May 26, 2006, the FDA granted accelerated approval for thalidomide in combination with dexamethasone for the treatment of newly diagnosed multiple myeloma patients. Thalidomide was introduced in the late 1950s as a sedative and for the treatment of morning sickness in pregnant women. The drug was withdrawn from the market in the early 1960s because reports of birth defects such as phocomelia were associated with its use. Thirty years later, it was established that this complication was secondary to inhibition of blood vessel growth in the development of fetal limb buds. However, thalidomide and its analogues (lenalidomide, CC-4047) have immunomodulatory, antiangiogenic, antiproliferative, and proapoptotic properties, which are responsible for antitumor action. Thalidomide also inhibits the inflammatory response by decreasing cyclooxygenase- 2 activity. ${ }^{59,60}$ Thalidomide is a potent inhibitor of TNF- $\alpha$ by inducing TNF- $\alpha$ mRNA degradation, ${ }^{61}$ and thereby decreasing the density of TNF- $\alpha$-induced adhesion molecules such as ICAM-I and VCAM-I. ${ }^{62}$ Thalidomide and its analogues reduce the 
expression of proangiogenic factors such as VEGF and thus prevent angiogenesis. ${ }^{63}$ Thalidomide also causes induction of NK cells and increase the levels of IL-2, IL-2 receptors, and IFN- $\gamma$, leading to tumor cell lysis and modulate the immune system to induce anticancer activity. ${ }^{64}$ The function of other cytokines, such as IL-6 and IL-12, are inhibited by thalidomide, along with its effect on the expression of cell adhesion molecules. ${ }^{65-67}$ Mechanisms of thalidomideinduced apoptosis include cell growth arrest at the G1 phase, downregulation of NFKB and apoptosis inhibitory protein (AIP), and activation of caspase $8 .{ }^{68}$

\section{Safety profile}

Thalidomide as a single agent in a daily dose up to $400 \mathrm{mg}$ was well tolerated. The main toxicities are dose-dependent neuropathy, constipation, anorexia, dry mouth, skin rash, and fatigue. Most of the toxicities were manageable and reversible.

\section{Clinical experience in melanoma}

In a phase II efficacy study of a regimen including TMZ, thalidomide, and whole brain radiation therapy (WBRT) in patients with brain (central nervous system [CNS]) metastases from melanoma, 39 patients received treatment and seven patients had stable CNS disease at 10 weeks. No patient exhibited a systemic response. Median time to progression was seven weeks and median OS was four months. Grade 3-4 side effects included deep venous thrombosis (3), pulmonary embolism (1), and CNS events (12). Eighteen (45\%) patients required admission for side effects (7) and/or symptomatic disease progression (11). The efficacy of TMZ, thalidomide, and WBRT in the treatment of CNS metastatic melanoma is low. ${ }^{69}$

A phase II study evaluated the efficacy and tolerability of DTIC in combination with thalidomide in metastatic melanoma patients. Fifteen patients were enrolled in the study. Of the 13 patients evaluable for response, one patient had a PR, three patients had SD, and nine patients had PD. No complete responses were seen. Grade III neutropenia, thrombocytopenia and nausea were attributed to DTIC. Grade III/IV constipation, peripheral neuropathy, fatigue, edema and rash were attributed to thalidomide. The addition of thalidomide to DTIC in metastatic melanoma yielded activity insufficient to proceed with additional trials of this combination. Thalidomide dose escalation beyond $200 \mathrm{mg} /$ day was limited by unacceptable toxicity. Therefore, this combination does not warrant further investigation. ${ }^{70}$
Phase II studies evaluating single-agent thalidomide in metastatic malignant melanoma, showed no objective response, but few cases of stable disease. Among 36 eligible patients, 35 were evaluable for response. SD is very rare in patients with brain metastases with or without any treatment. Four patients (11\%) had stable brain metastases for more than four months on thalidomide, providing some indication of thalidomide activity in the CNS. The responding patient in the study progressed during treatment, with concomitant disease control in peripheral manifestations. This demonstrates thalidomide's activity in peripheral tumor manifestations. $^{71}$

In a phase II trial evaluating efficacy of pegylated INF and thalidomide in melanoma, pegylated INF was administered at a dose of $0.5 \mu \mathrm{g} / \mathrm{kg}$ subcutaneously weekly and thalidomide $200 \mathrm{mg}$ orally daily. Eighteen patients were enrolled in this trial. Severe (grade 4) toxicities observed were anemia in two patients and thrombocytopenia in one patient. No ORs were noted. Three patients demonstrated $\mathrm{SD}$. The regimen of pegylated INF and thalidomide was well tolerated. The combination, however, failed to demonstrate clinical efficacy in pretreated metastatic malignant melanoma. $^{72}$

\section{Conclusion}

Melanoma continues to be a cancer that is exquisitely resistant to treatment. Chemotherapy and immunotherapy have thus far failed to make an impact on survival in the metastatic setting while immunotherapy leads to a modest improvement in survival in the adjuvant setting. Elucidation of the molecular mechanisms of carcinogenesis in melanoma have expanded the horizon of opportunity to alter the natural history of the disease. Multiple signal transduction pathways seem to be aberrant and drugs that target them have been and continue to be in development. In this review we have presented data on the most promising agents in development. However, it is important to note that all of these agents have been used singly or occasionally in combination with chemotherapy. It has become quite apparent that the inhibition of one pathway can lead to the upregulation of other related or redundant pathways. This may negatively affect its activity or likely lead to resistance therefore combination of therapies utilizing these agents seems to be the most promising approach in the near future. Mutational analysis of melanoma tissue can identify patients more likely to benefit from targeted agents. However, more comprehensive molecular profiling will refine our ability to rationally individualize the treatment of melanoma. 


\section{Disclosures}

The authors report no conflicts of interest in this work.

\section{References}

1. Hood JD, Bednarski M, Frausto R, et al. Tumor regression by targeted gene delivery to the neovasculature. Science. 2002;296: 2404-2407.

2. Gray-Schopfer V, Wellbrock C, Marais R. Melanoma biology and new targeted therapy. Nature. 2007;445:851-857.

3. Satyamoorthy K, Li G, Gerrero MR, et al. Constitutive mitogenactivated protein kinase activation in melanoma is mediated by both BRAF mutations and autocrine growth factor stimulation. Cancer Res. 2003;63:756-759.

4. Lyons JF, Wilhelm S, Hibner B, Bollag G. Discovery of a novel Raf kinase inhibitor. Endocr Relat Cancer. 2001;8:219-225.

5. Wilhelm S, Chien DS. BAY 43-9006: Preclinical data. Curr Pharm Des. 2002;8:2255-2257.

6. Wilhelm SM, Carter C, Tang L, et al. BAY 43-9006 exhibits broad spectrum oral antitumor activity and targets the raf/mek/erk pathway and receptor tyrosine kinases involved in tumor progression and angiogenesis. Cancer Res. 2004;64:7099-7109.

7. Panka DJ, Wang W, Atkins MB, Mier JW. The Raf inhibitor BAY 43-9006 (Sorafenib) induces caspase-independent apoptosis in melanoma cells. Cancer Res. 2006;66:1611-1619.

8. Rahmani M, Davis EM, Bauer C, Dent P, Grant S. Apoptosis induced by the kinase inhibitor BAY 43-9006 in human leukemia cells involves down-regulation of Mcl-1 through inhibition of translation. J Biol Chem. 2005;280:35217-35227.

9. Yu C, Bruzek LM, Meng XW, et al. The role of Mcl-1 downregulation in the proapoptotic activity of the multikinase inhibitor BAY 43-9006. Oncogene. 2005;24:6861-6869.

10. Graells J, Vinyals A, Figueras A, et al. Overproduction of vegf concomitantly expressed with its receptors promotes growth and survival of melanoma cells through mapk and pi3k signaling. J Invest Dermatol. 2004;123:1151-1161.

11. Escudier B, Eisen T, Stadler WM, et al. Sorafenib in advanced clear-cell renal-cell carcinoma. $N$ Engl J Med. 2007;356:125-134.

12. Strumberg D, Clark JW, Awada A, et al. Safety, pharmacokinetics, and preliminary antitumor activity of sorafenib: a review of four phase I trials in patients with advanced refractory solid tumors. Oncologist. 2007;12:426-437.

13. Ratain MJ, Eisen T, Stadler WM, et al. Phase II placebo-controlled randomized discontinuation trial of sorafenib in patients with metastatic renal cell carcinoma. J Clin Oncol. 2006;24:2505-2512.

14. Chatelut E, Delord JP, Canal P. Toxicity patterns of cytotoxic drugs. Invest New Drugs. 2003;21:141-148.

15. Hauschild A, Agarwala SS, Trefzer U, et al. Results of a phase III, randomized, placebo-controlled study of sorafenib in combination with carboplatin and paclitaxel as second-line treatment in patients with unresectable stage III or stage IV melanoma. J Clin Oncol. 2009;27(17):2823-2830.

16. Eisen T, Ahmad T, Marais R, et al. Phase I trial of sorafenib (BAY 43-9006) combined with dacarbazine (DTIC) in patients with metastatic melanoma [abstract]. Eur J Cancer Suppl. 2005;3:349.

17. Eisen T, Marais R, Affolter A, et al. An open-label phase ii study of sorafenib and dacarbazine as first line therapy in patients with advanced melanoma [abstract]. J Clin Oncol. 2007;25:8529.

18. McDermott DF, Sosman JA, Gonzalez R, et al. Double-blind randomized phase II study of the combination of sorafenib and dacarbazine in patients with advanced melanoma: A report from the 11715 study group. J Clin Oncol. 2008;26:2178-2185.

19. Amaravadi R, Schuchter LM, McDermott DF, et al. Updated results of a randomized phase ii study comparing two schedules of temozolomide in combination with sorafenib in patiets with advanced melanoma [abstract]. J Clin Oncol. 2007;25:8527.
20. Soria J, Lazar V, Lassau N, et al. Sorafenib (S) and dacarbazine (D) combination in patients (pts) with advanced malignant solid tumors: Phase I study with tumor biopsy genomic analysis and dynamic contrast enhanced ultrasonography (DCE-US) [abstract]. J Clin Oncol. 2007;25:3556

21. Flaherty K, Puzanov I, Sosman J, et al. Phase I study of PLX4032: Proof of concept for V600E BRAF mutation as a therapeutic target in human cancer [abstract]. J Clin Oncol. 2009;27(Suppl 15):9000.

22. Tarhini AA, Kirkwood JM. Oblimersen in the treatment of metastatic melanoma. Future Oncol. 2007;3:263-271.

23. Genta I. Genta's lead antisense drug reported active in malignant melanoma new data on antisense mechanism and clinical response reported in leading journal. Media Release: 2000.

24. Jansen B, Wacheck V, Heere-Ress E, et al. Chemosensitisation of malignant melanoma by BCL2 antisense therapy. Lancet. 2000;356: $1728-1733$.

25. Pavlick AC, Escalon J, Madden K, et al. A phase I/II study to determine the feasibility and efficacy of the triple combination of oblimersen (OBL), abraxane (ABX), and temozolomide (TMZ) in metastatic melanoma and normal LDH. J Clin Oncol. 2008;26(Suppl):9027.

26. Bedikian AY, Millward M, Pehamberger H, et al. Bcl-2 antisense (oblimersen sodium) plus dacarbazine in patients with advanced melanoma: the Oblimersen Melanoma Study Group. J Clin Oncol. 2006;24:4738-4745.

27. Lev DC, Onn A, Melinkova VO, et al. Exposure of melanoma cells to dacarbazine results in enhanced tumor growth and metastasis in vivo. J Clin Oncol. 2004;22:2092-2100.

28. Jain RK. Normalizing tumor vasculature with anti-angiogenic therapy: A new paradigm for combination therapy. Nature Med. 2001;7:987-989.

29. Nör JE, Christensen J, Mooney DJ, Polverini PJ. Vascular endothelial growth factor (VEGF)-mediated angiogenesis is associated with enhanced endothelial cell survival and induction of Bcl-2 expression. Am J Pathol. 1999;154:375-384.

30. Masood R, Cai J, Zheng T, Smith DL, Hinton DR, Gill PS. Vascular endothelial growth factor (VEGF) is an autocrine growth factor for VEGF receptor-positive human tumors. Blood. 2001;98:1904-1913.

31. Lacal PM, Failla CM, Pagani E, et al. Human melanoma cells secrete and respond to placenta growth factor and vascular endothelial growth factor. J Invest Dermatol. 2000;115:1000-1007.

32. Varker KA, Biber JE, Kefauver C, et al. A randomized phase 2 trial of bevacizumab with or without daily low-dose interferon alfa-2b in metastatic malignant melanoma. Ann Surg Oncol. 2007;14:2367-2376.

33. Sandler A, Gray R, Perry MC, et al. Paclitaxel-carboplatin alone or with bevacizumab for non-small-cell lung cancer. $N$ Engl $J$ Med. 2006;355:2542-2550.

34. Kabbinavar FF, Hambleton J, Mass RD, Hurwitz HI, Bergsland E, Sarkar S. Combined analysis of efficacy: The addition of bevacizumab to fluorouracil/leucovorin improves survival for patients with metastatic colorectal cancer. J Clin Oncol. 2005;23:3706-3712.

35. Perez DG, Suman VJ, Fitch TR, et al. Phase 2 trial of carboplatin, weekly paclitaxel, and biweekly bevacizumab in patients with unresectable stage IV melanoma: a North Central Cancer Treatment Group study, N047 A. Cancer. 2009;115:119-127.

36. Gera JF, Mellinghoff IK, Shi Y, et al. AKT activity determines sensitivity to mammalian target of rapamycin (mTOR) inhibitors by regulating cyclin D1 and c-myc expression. J Biol Chem. 2004;279: 2737-2746.

37. Neshat MS, Mellinghoff IK, Tran C, et al. Enhanced sensitivity of PTEN-deficient tumors to inhibition of FRAP/mTOR. Proc Natl Acad Sci U S A. 2001;98:10314-10319.

38. Schmelzle T, Hall MN. TOR, a central controller of cell growth. Cell. 2000;103:253-262.

39. Huang S, Houghton PJ. Mechanisms of resistance to rapamycins. Drug Resist Updat. 2001;4:378-391.

40. Raymond E, Alexandre J, Depenbrock H, et al. CCI-779, a rapamycin analog with antitumor activity: A phase I study utilizing a weekly schedule [abstract]. Proc Am Soc Clin Oncol. 2000;19:187. 
41. Peralba JM, DeGraffenried L, Friedrichs W, et al. Pharmacodynamic evaluation of CCI-779, an inhibitor of mTOR, in cancer patients. Clin Cancer Res. 2003;9:2887-2892.

42. Dutcher JP, Hudes G, Motzer R, et al. Preliminary report of a phase 1 study of intravenous (IV) CCI-779 given in combination with interferon(IFN) to patients with advanced renal cell carcinoma (RCC) [abstract]. Proc Am Soc Clin Oncol. 2003;22:213.

43. Adams J, Palombella VJ, Sausville EA, et al. Proteasome inhibitors: A novel class of potent and effective antitumor agents. Cancer Res. 1999;59:2615-2622.

44. Ling YH, Liebes L, Jiang JD, et al. Mechanisms of proteasome inhibitor PS-341-induced $\mathrm{G}_{2}$-M-Phase arrest and apoptosis in human non-small cell lung cancer cell lines. Clin Cancer Res. 2003;9:1145-1154.

45. Nagata Y, Anan T, Yoshida T, et al. The stabilization mechanism of mutant-type $\mathrm{p} 53$ by impaired ubiquitination: The loss of wild-type p53 function and the hsp90 association. Oncogene. 1999;18:6037-6049.

46. An WG, Hwang SG, Trepel JB, Blagosklonny MV. Protease inhibitorinduced apoptosis: Accumulation of wt p53, p21waf1/cip1, and induction of apoptosis are independent markers of proteasome inhibition. Leukemia. 2000;14:1276-1283.

47. Alessandrini A, Chiaur DS, Pagano M. Regulation of the cyclindependent kinase inhibitor p27 by degradation and phosphorylation. Leukemia. 1997;11:342-345.

48. Palombella VJ, Conner EM, Fuseler JW, et al. Role of the proteasome and nf-kappab in streptococcal cell wall-induced polyarthritis. Proc Natl Acad Sci U S A. 1998;95:15671-15676.

49. Read MA, Neish AS, Luscinskas FW, Palombella VJ, Maniatis T, Collins T. The proteasome pathway is required for cytokine-induced endothelial-leukocyte adhesion molecule expression. Immunity. 1995;2:493-506.

50. Nix D, Pien C, Newman R, et al. Clinical development of a proteasome inhibitor, PS-341, for the treatment of cancer. Proc Am Soc Clin Oncol. 2001;20:86a.

51. Markovic SN, Geyer SM, Dawkins F, et al. A phase II study of bortezomib in the treatment of metastatic malignant melanoma. Cancer. 2005;103:2584-2589.

52. Kendra KL, Lesinski GB, Olencki TE, Carson W. A phase I study of bortezomib and interferon-alpha-2b in patients with metastatic melanoma [abstract]. J Clin Oncol. 2008.

53. Roberts JD, Ernstoff MS, Birdsell C. Phase I trial of dacarbazine and bortezomib in melanoma and soft tissue sarcoma [abstract]. J Clin Oncol. 2006.

54. Dal Lago L, D'Hondt V, Awada A. Selected combination therapy with sorafenib: A review of clinical data and perspectives in advanced solid tumors. Oncologist. 2008;13:845-858.

55. Haass NK, Sproesser K, Nguyen TK, et al. The mitogen-activated protein/extracellular signal-regulated kinase kinase inhibitor AZD6244 (ARRY-142886) induces growth arrest in melanoma cells and tumor regression when combined with docetaxel. Clin Cancer Res. 2008; 14:230-239.

56. Smalley KS, Haass NK, Brafford PA, Lioni M, Flaherty KT, Herlyn M. Multiple signaling pathways must be targeted to overcome drug resistance in cell lines derived from melanoma metastases. Mol Cancer Ther. 2006;5:1136-1144.
57. Adjei AA, Cohen RB, Franklin W, et al. Phase I pharmacokinetic and pharmacodynamic study of the oral, small-molecule mitogen-activated protein kinase kinase 1/2 inhibitor AZD6244 (ARRY-142886) in patients with advanced cancers. J Clin Oncol. 2008;26:2139-2146.

58. Dummer R, Robert C, Chapman PB, et al. AZD6244 (ARRY-142886) vs temozolomide (TMZ) in patients (pts) with advanced melanoma: An open-label, randomized, multicenter, phase II study [abstract ]. J Clin Oncol. 2008;26(Suppl):9033.

59. Rajkumar SV. Thalidomide in the treatment of multiple myeloma. Expert Rev Anticancer Ther. 2001;1:20-28.

60. Quilitz R. Thalidomide in oncology: The peril and the promise. Cancer Control. 1999;6:483-495.

61. Moreira AL, Sampaio EP, Zmuidzinas A, Frindt P, Smith KA, Kaplan G. Thalidomide exerts its inhibitory action on tumor necrosis factor alpha by enhancing mRNA degradation. J Exper Med. 1993;177: 1675-1680.

62. Geitz H, Handt S, Zwingenberger K. Thalidomide selectively modulates the density of cell surface molecules involved in the adhesion cascade. Immunopharmacology. 1996;31:213-221.

63. Gupta D, Treon SP, Shima Y, et al. Adherence of multiple myeloma cells to bone marrow stromal cells upregulates vascular endothelial growth factor secretion: therapeutic applications. Leukemia. 2001;15: 1950-1961.

64. Davies FE, Raje N, Hideshima T, et al. Thalidomide and immunomodulatory derivatives augment natural killer cell cytotoxicity in multiple myeloma. Blood. 2001;98:210-216.

65. Meierhofer C, Wiedermann CJ. New insights into the pharmacological and toxicological effects of thalidomide. Curr Opin Drug Discov Devel. 2003;6:92-99.

66. Franks ME, Macpherson GR, Figg WD. Thalidomide. Lancet. 2004; 363:1802-1811.

67. Meierhofer C, Dunzendorfer S, Wiedermann CJ. Theoretical basis for the activity of thalidomide. BioDrugs. 2001;15:681-703.

68. Mitsiades N, Mitsiades CS, Poulaki V, et al. Apoptotic signaling induced by immunomodulatory thalidomide analogs in human multiple myeloma cells: therapeutic implications. Blood. 2002;99:4525-4530.

69. Atkins MB, Sosman JA, Agarwala S, et al. Temozolomide, thalidomide, and whole brain radiation therapy for patients with brain metastasis from metastatic melanoma: A phase II cytokine working group study. Cancer. 2008;113:2139-2145.

70. Ott PA, Chang JL, Oratz R, et al. Phase II trial of dacarbazine and thalidomide for the treatment of metastatic melanoma. Chemotherapy. 2009;55:221-227.

71. Vestermark LW, Larsen S, Lindelov B, Bastholt L. A phase II study of thalidomide in patients with brain metastases from malignant melanoma. Acta Oncol. 2008;47:1526-1530.

72. Vaishampayan UN, Heilbrun LK, Marsack C, Smith DW, Flaherty LE. Phase II trial of pegylated interferon and thalidomide in malignant metastatic melanoma. Anticancer Drugs. 2007;18:1221-1226.

\section{Publish your work in this journal}

Biologics: Targets \& Therapy is an international, peer-reviewed journal focusing on the patho-physiological rationale for and clinical application of Biologic agents in the management of autoimmune diseases, cancers or other pathologies where a molecular target can be identified. This journal is indexed on PubMed Central, CAS,

\section{Dovepress}

EMBase, Scopus and the Elsevier Bibliographic databases. The manuscript management system is completely online and includes a very quick and fair peer-review system, which is all easy to use. Visit http://www.dovepress.com/testimonials.php to read real quotes from published authors. 